\title{
Influencia de la Longitud, la Complejidad y la Inferencia en la Resolución de Pruebas de Comprensión Lectora Inferencial
}

\author{
Effects of Length, Complexity, and Inference in the Resolution \\ of Inferential Reading Comprehension Tests
}

\author{
Alejandra Platas-García *12 \\ J. Martín Castro-Manzano ${ }^{2}$ \\ Verónica Reyes-Meza ${ }^{3}$ \\ Irene Gaona-Gordillo ${ }^{3}$ \\ ${ }^{1}$ Benemérita Universidad Autónoma de Puebla (BUAP), México \\ ${ }^{2}$ Universidad Popular Autónoma del Estado de Puebla (UPAEP), México \\ ${ }^{3}$ Universidad Autónoma de Tlaxcala (UATx), México
}

\begin{abstract}
Este trabajo surge a partir de nuestra experiencia docente a nivel superior con las tareas de revisión y elaboración de pruebas de opción múltiple para medir comprensión lectora. Nuestros objetivos son dos: 1) determinar si las variables de longitud, complejidad e inferencia de una prueba de comprensión lectora inferencial tienen efectos en el desempeño de los estudiantes y, consecuentemente, 2) argumentar a favor de la elaboración de pruebas que promuevan o incluyan el uso de inferencias no-deductivas en adición a las deductivas. Para lograr estos objetivos llevamos a cabo un estudio con 187 estudiantes universitarios a los que les aplicamos una prueba de comprensión lectora inferencial de opción múltiple. Los datos obtenidos fueron analizados con un modelo lineal generalizado mixto. Entre los resultados generales encontramos que los estudiantes tienen un mejor desempeño en la prueba de comprensión lectora cuando se les solicita realizar inferencias no-deductivas y los textos son complejos y largos; mientras que su desempeño decrece cuando se les solicita realizar inferencias deductivas y los textos son simples y cortos. Estos resultados parecen soportar la tesis de que existe un efecto de las características de las pruebas en la comprensión lectora de los estudiantes, lo cual, a su vez, nos permite sugerir que el diseño de pruebas de comprensión lectora podría enriquecerse tomando en cuenta un concepto más incluyente de inferencia que sería consistente con el concepto de racionalidad acotada.
\end{abstract}

Palabras clave: Evaluación de estudiantes; Razonamiento, Deducción; Tests de lectura; Tests de elección múltiple; Comprensión lectora; Inferencias.

Our interest in this research comes from our experience at reviewing and developing multiple-choice tests in order to measure reading comprehension skills at an undergraduate level. We have two goals: 1) to determine if the variables of length, complexity, and inference, of a reading comprehension test have an effect over students performance and, consequently, 2) to argue in favour of tests that promote or include non-deductive inference in addition to deductive inference as a parameter of their design. To reach these goals we have applied an inferential reading comprehension test to a group of 187 undergraduate students. The obtained data was analysed with a generalized linear mixed model. Among the general results, we find that students perform better when the test requires them to carry out a non-deductive inference over complex and long texts; whereas their performance decreases when the test calls for a deductive inference over simple and short texts. These results seemingly support the claim that there is an influence of the properties of the test over the students reading comprehension, which, in turn, allows us to suggest that the design of reading comprehension tests might be enriched by taking into account a more inclusive concept of inference consistent with the notion of bounded rationality.

Keywords: Student evaluation; Reasoning; Deduction; Reading tests; Multiple choice tests; Reading comprehension; Inferences.

\begin{tabular}{lll}
\hline *Contacto: aplatasg@gmail.com & & \\
issn: 1989-0397 & Recibido: & 24 de noviembre de 2017 \\
www.rinace.net/riee/ & $1^{\text {a Evaluación: }} 18$ de diciembre de 2017 \\
https://revistas.uam.es/riee & Aceptado: & 30 de enero de 2018
\end{tabular}




\section{Introducción}

Este trabajo surge a partir de nuestra experiencia docente a nivel superior con las tareas de revisión y elaboración de pruebas de comprensión lectora, específicamente, pruebas de opción múltiple en las que se presenta un texto a partir del cual se realizan preguntas y se ofrecen, comúnmente, tres o cuatro opciones de respuesta y sólo una de ellas es correcta. En el contexto de este tipo de pruebas creemos que es pertinente reconsiderar las características de su diseño, porque podría existir una influencia del mismo en el desempeño de los estudiantes; lo que, de ser cierto, nos podría ofrecer elementos de juicio para argumentar a favor de la elaboración de pruebas que promuevan una comprensión inferencial enriquecida, dado que, por ejemplo, como afirman Guerra y Guevara (2013), los problemas de comprensión lectora en estudiantes universitarios residen en la dificultad de identificar "la o las ideas principales y [...] el uso apropiado de la información para hacer inferencias y realizar un análisis crítico sobre la base de lo leído” (p. 278).

Motivados por estas consideraciones, hemos analizado ejemplos de pruebas de comprensión lectora de opción múltiple con el propósito de revisar algunas de sus propiedades estructurales: características materiales que comparten (longitud), cualidades que pueden ser distinguidas (complejidad) y el tipo de inferencia que propician (deductiva, no-deductiva). De acuerdo al modelo de van Dijk (1992), las propiedades estructurales de un texto son tres: 1) la microestructura que engloba los enunciados y las secuencias de enunciados, 2) la macroestructura que proporciona la información que dirigirá la interpretación de las palabras y los enunciados, y que representa la coherencia global del texto, y 3) la superestructura que es un tipo de esquema abstracto que establece el orden general al cual el texto se adapta. Dado este modelo, en esta colaboración trabajamos una analogía entre los atributos estructurales de los textos y las propiedades estructurales de las pruebas previamente mencionadas: 1) la microestructura se asocia con la longitude, 2) la macroestructura con la Complejidad, y 3) la superestructura con los tipos de inferencia.

Así pues, este estudio tiene dos objetivos: 1) conocer si la longitud (corto, largo), la complejidad (simple, complejo) y el tipo de inferencia (deductiva, no-deductiva) de una prueba de comprensión lectora inferencial influyen en el desempeño de los estudiantes y, consecuentemente y 2) argumentar a favor de la elaboración de pruebas que promuevan inferencias no-deductivas además de las deductivas. La organización del trabajo es la siguiente. En la primera sección ofrecemos una breve caracterización del concepto de comprensión lectora que asumimos como marco teórico general. Posteriormente, en la segunda, describimos las propiedades estructurales de longitud, complejidad e inferencia. En la tercera, analizamos una prueba de comprensión lectora a la luz de sus características de superestructura. En la cuarta, exponemos el concepto de inferencia extendida y racionalidad acotada. En la quinta, mostramos la metodología que seguimos. En la sexta, los resultados obtenidos. Finalmente, en la séptima, presentamos una breve discusión y algunas conclusiones.

\section{Comprensión lectora}

Existen tres enfoques principales sobre el proceso de comprensión lectora, a saber: 1) el modelo abajo-arriba (bottom up) que considera que el proceso de comprensión procede de la percepción visual, el reconocimiento de letras y palabras, la agrupación de palabras para 
formar proposiciones y así sucesivamente, cada operación es requerida para que se realice la siguiente; 2) el modelo arriba-abajo (top down) para el que los aspectos perceptivo-visuales están subordinados a los conocimientos semánticos y sintácticos del lector; y 3) los modelos interactivos para los que el proceso de comprensión está dirigido en forma interactiva tanto por el texto como por el conocimiento del lector (Acosta, 2009). En esta investigación, la comprensión lectora es entendida bajo el tercer enfoque por lo que se define como "un proceso a través del cual el lector elabora un significado en su interacción con el texto" (Pérez Zorrilla, 2005, p. 123; cf. Guerra y Guevara, 2017, p. 79).

En el proceso de comprensión lectora, el lector aporta sus esquemas de conocimiento (que son fruto tanto de sus experiencias como de aprendizajes previos) con el fin de integrar los datos nuevos incluidos en el texto; en este proceso, los esquemas del lector pueden sufrir modificaciones y enriquecimientos continuos (Solé, 1987). (Sobre los esquemas véase: Anderson y Pearson, 1987; Intraversato, 2013; Salazar, 2006).

Como se puede inferir, el proceso de comprensión lectora requiere que el lector posea un conjunto articulado de conocimientos y habilidades que van más allá de una simple decodificación o comprensión literal del texto (esto es consistente con el modelo de García, 1993, que propone niveles ordenados: decodificación, comprensión literal, comprensión inferencial y metacomprensión), por esto, es un proceso activo e interactivo de construcción de significados en el que están implicados el lector, el texto y el contexto (Giovannini y Rosa, 2015).

En este trabajo asumimos un nivel de comprensión lectora de nivel inferencial siguiendo la clasificación de García (1993), para quien la elaboración de inferencias forma parte del procesamiento textual, el cual está conformado por representaciones, por la integración de enunciados, el conocimiento del mundo por parte de un lector (lo cual es consistente con el concepto de conocimientos enciclopédicos en la propuesta de Intraversato, 2013), "las inferencias según los esquemas cognitivos que el mismo lector posee y su interpretación del texto" (García, 1993, p. 94).

Por estas razones la comprensión lectora representa una tarea fundamental en la formación académica y, por tanto, en el contexto educativo; por ello los docentes y las instituciones educativas se interesan por conocer cuál es el nivel de comprensión lectora que poseen los estudiantes. Sin embargo, la medición de este nivel de comprensión no es transparente, por lo que para obtener evidencias es necesario realizar evaluaciones indirectas (Consejo de Europa, 2002), ya que como afirma Pérez Zorrilla (2005), "dado que no se puede observar directamente la comprensión lectora, hay que pedirle al alumno que realice algún tipo de tarea que indique el grado de comprensión alcanzado” (p. 128). Esta evaluación indirecta se suele llevar a cabo mediante pruebas en las que se solicita al estudiante que realice tareas como marcar casillas, completar frases, contestar preguntas o elegir una opción correcta de entre una serie finita de alternativas. Dentro de este conjunto de pruebas, una que es utilizada frecuentemente para evaluar la comprensión lectora es la prueba de opción múltiple. En la siguiente sección revisamos la estructura de este tipo de pruebas trabajando la analogía con el modelo de van Dijk. 


\section{Propiedades estructurales de las pruebas}

Para estudiar los atributos de micro (longitud), macro (complejidad) y superestructura (inferencia) hemos diseñado una prueba de comprensión lectora inferencial de opción múltiple que usamos como instrumento para esta investigación. En la sección de metodología ofrecemos más detalles de la prueba.

\subsection{Longitud}

Si bien esta característica es importante para definir la complejidad de un texto, en este estudio la hemos distinguido de la complejidad per se por razones que mencionaremos en el siguiente apartado. Para definir la longitud del texto se pueden emplear distintos criterios, por ejemplo: el número de palabras (cf. Rice, 1994, para quien un texto corto es de 142 palabras; mientras que para Bell, 2001, un texto corto es de 300) o el número de páginas (cf. Mangen, Walgermo y Brønnick, 2013, para quienes cuatro páginas forman un texto corto). El criterio empleado en esta investigación fue el número de líneas que tiene cada tex to asumiendo que: 1) los márgenes y el tamaño de las fuentes son fijos e invariables y 2) la información en los textos no es redundante. De esta forma, en esta investigación, un texto es corto, si el número de líneas es menor o igual a 4; y es largo, si el número de líneas es mayor o igual a 5 y menor o igual a 8 . Este es un criterio viable para el análisis de las características del texto presentado, ya que si los textos fueran de una extensión mayor a los rangos mencionados, harían de la prueba un instrumento inviable en términos del tiempo utilizado para su aplicación.

\subsection{Complejidad}

De acuerdo con Ferrari (2003), para conocer la dificultad de un texto existen dos criterios, uno cuantitativo, la legibilidad, y otro cualitativo, la comprensibilidad. Con respecto al primero, la autora afirma que las investigaciones sobre legibilidad tienen como objetivo la construcción de funciones matemáticas que tomen como entrada un texto y asignen un índice numérico que ilustre su dificultad, considerando como factores de complejidad las frases largas y las palabras poco comunes, por ejemplo; sin embargo, estas funciones no necesariamente toman en cuenta las competencias y conocimientos individuales del lector; del mismo modo, excluyen otros aspectos importantes de un texto, como la estructura lógica o la presencia de tablas y figuras. Por esto último, Ferrari (2003) sugiere que la legibilidad no puede considerarse como un índice preciso de la dificultad de un texto.

Además, sobre la misma legibilidad, Pallotti (2013) afirma que este tipo de funciones matemáticas se basan en el cálculo de la longitud media de los enunciados y de las palabras: textos con enunciados largos y palabras largas tendrán un valor de legibilidad más bajo que los textos con enunciados cortos y palabras cortas. Sin embargo, este indicador no basta para afirmar que un texto es simple o complejo, puesto que es necesario acudir al segundo criterio, la comprensibilidad, ya que un texto que contiene únicamente enunciados cortos y palabras cortas puede resultar obscuro o poco asequible debido a posibles lagunas informativas.

Por esto, el segundo criterio, la comprensibilidad, parece ser más útil para el análisis de la dificultad de un texto, pues los estudios de comprensibilidad no tienen por objetivo obtener índices numéricos, sino explorar y utilizar metodologías de análisis de dificultad de un texto como las que ofrece la lingüística textual, que ha identificado dos propiedades 
fundamentales de un texto, a saber: la cohesión y la coherencia. Entendiéndose por cohesión la serie de mecanismos que aseguran la unión entre las partes de los textos a nivel superficial, es decir, el respeto de las relaciones formales entre los enunciados (coordinación y subordinación), las referencias temporales, el orden de las palabras y el uso de conectores como adverbios o conjunciones que sirven para unir explícitamente dos enunciados; la coherencia, por otro lado, está relacionada con los procesos lógicos y conceptuales que permiten la producción de sentido (Ferrari, 2003). En suma, mientras la legibilidad es una característica intrínseca del texto y es medible, la comprensibilidad es una característica proporcional, pues depende de la distancia de los conocimientos y competencias del lector al texto, a su estructura, a su contenido (Zambelli, 2014).

Pues bien, dado que la clasificación de un texto como simple o complejo no es una tarea sencilla y no basta con una métrica de legibilidad o longitud, optamos por tomar como criterio de complejidad el juicio de un grupo de expertos asumiendo la noción de comprensibilidad y tomando en cuenta los siguientes criterios: verbos, vocabulario, redacción, extensión y tema. De esta forma, dado un universo de 7 lectores expertos (escritores, editores, docentes de español para extranjeros), un texto es simple, si 4 o más expertos etiquetan un texto como "simple"; un texto es complejo, si 4 o más expertos lo etiquetan como "complejo".

\subsection{Inferencia}

Pallotti (2013) afirma que en el proceso activo de la comprensión de un texto el lector busca darle sentido a lo que lee revisando la coherencia entre el contenido del texto y sus conocimientos previos; además, dado que un texto no puede decirlo todo y mucha información se da por sobreentendida, el lector busca unidad a través de una serie de inferencias con las cuales llena los vacíos e integra la información en un todo coherente; del mismo modo, extrae las consecuencias de lo que está leyendo, usando ocasionalmente su conocimiento general (p. 3). La inferencia, por tanto, es un proceso relacionado con la comprensión lectora en el que a partir de la aceptación de ciertas información (premisas) se llega a la aceptación de cierta información (conclusión) (Copi y Cohen, 2007; Cook, 2009).

Una inferencia puede ser de tres distintos tipos, dependiendo de la relación entre las premisas y la conclusión. En algunas inferencias la conclusión no añade información nueva a lo que se ha expresado en las premisas; mientras que en otros tipos de inferencia la conclusión sí puede añadir información nueva. Esta distinción básica induce la división operacional entre inferencias deductivas y no-deductivas (inducción y abducción) que utilizamos para este estudio.

a) Deducción. En sentido estricto, una deducción se define como una secuencia finita de enunciados dentro de un sistema formal donde cada enunciado en la secuencia es un axioma, una suposición o el resultado de la aplicación de una regla de inferencia a uno o varios enunciados precedentes. El enunciado final es la conclusión del argumento que ha sido derivado y cada suposición es una premisa del argumento derivado (Cook, 2009). De manera informal, en una deducción la información está completa y por ende la conclusión no agrega nada nuevo a lo que ya está expresado en las premisas (Rodríguez Rodríguez, 2005). Por ello, en una inferencia deductiva es imposible que las premisas sean verdaderas y la conclusión sea falsa (Cook, 2009). Un ejemplo típico de inferencia deductiva sería el siguiente: 


\section{Todos los hombres son mortales. 2. Sócrates es un hombre. \\ C. Por tanto, Sócrates es mortal.}

b) Inducción. El concepto más difundido de inducción afirma que esta es una inferencia que va de lo particular a lo general. Como este concepto es muy ambiguo, se ha desarrollado un concepto más claro: una inducción es una inferencia que permite extraer conclusiones a partir de información incompleta dado cierto grado de soporte (Hawthorne, 2014). El grado de soporte que proveen las premisas ofrece la base conceptual que sostiene a la conclusión, pero este grado de soporte no es deductivo, no implica necesidad, sino probabilidad; por ello, a diferencia de la inferencia deductiva, la inductiva bien podría partir de premisas verdaderas y llegar a conclusiones falsas (Cook, 2009). Consideremos un ejemplo de inferencia inductiva adaptado de Okasha (2002):

1. Los cinco primeros huevos de esta caja estaban descompuestos.

2. Los huevos en la caja tienen la misma fecha de caducidad.

C. Por tanto, muy probablemente, el sexto huevo estará descompuesto también.

c) Abducción. Una abducción es una inferencia cuyo producto es una explicación, con cierta estructura inferencial, que se dispara por un hecho que necesita explicarse (Aliseda, 1998, p. 10-11). Como la inducción, la abducción no requiere criterios de necesidad sino de probabilidad y es también un tipo de inferencia sintética. Según el planteamiento de Aliseda (1997, 1998), la diferencia entre inducción y abducción consiste en que mientras la última es una inferencia que parte de un hecho particular para buscar su explicación, la primera parte de una serie de observaciones para llegar a enunciados generales. A continuación mostramos un ejemplo de abducción adaptado de Okasha (2002):

\section{El queso en la alacena desapareció, excepto por unas pocas migajas. \\ 2. Anoche se oyeron ruidos que provenían de la alacena. \\ C. Luego, seguramente, un ratón se comió el queso.}

Además de las propiedades estructurales previamente descritas, es importante hacer el siguiente par de aclaraciones antes de continuar con nuestra exposición. Primero, es posible clasificar los textos como continuos o no-continuos por su estructura interna y el procesamiento requerido para su comprensión (Serrano, Vidal-Abarca y Ferrer, 2017); y segundo, es posible dividirlos en narrativos, descriptivos, expositivos y argumentativos de acuerdo a su tipología textual (Sánchez Lobato, 2007). Dadas estas consideraciones, para el diseño de nuestra prueba hemos elegido trabajar con textos continuos-descriptivos. Continuos, porque este tipo de textos presentan un orden secuencial determinado que ayuda a los estudiantes a entender las ideas "relacionándolas unas con otras mediante inferencias, hasta formar una representación mental coherente" (Serrano et al., 2017, p. 283); descriptivos, porque "presentan con claridad y rigor los rasgos característicos de personas, animales, objetos, lugares, fenómenos o situaciones” (Sánchez Lobato, 2007, p. $61)$. 


\section{Análisis de una prueba de comprensión lectora a la luz de los tipos de inferencia}

Si bien las propiedades estructurales de longitud y complejidad no son triviales, parece que son más fáciles de identificar, a diferencia de la propiedad de inferencia. Por ello, en esta sección ilustramos la importancia de reconsiderar la propiedad de inferencia a través del análisis de la sección de comprensión lectora del examen Diplomas de Español como Lengua Extranjera (DELE) en la prueba de opción múltiple a partir de los ejemplos disponibles en el sitio web del Instituto Cervantes para los niveles del Marco común europeo de referencia para las lenguas (MCERL): A1, A2, B1, B2, C1. El DELE es un examen que acredita el grado de competencia y dominio del idioma español y que otorga el Ministerio de Educación, Cultura y Deporte de España. El Instituto Cervantes se encarga de organizar las convocatorias de los exámenes, mientras que la Universidad de Salamanca, en España, se dedica a la elaboración, corrección y evaluación final de los exámenes. En el cuadro 1 presentamos algunos casos representativos que permiten apreciar el tipo de inferencia que solicitan las versiones consultadas.

Cuadro 1. Ejemplos de inferencias solicitadas en una prueba de comprensión de lectura del examen DELE

\begin{tabular}{|c|c|c|c|}
\hline $\begin{array}{l}\text { DATOS DEL } \\
\text { EXAMEN } \\
\text { DELE } \\
\end{array}$ & $\begin{array}{c}\text { FRAGMENTO DEL TEXTO } \\
\text { PARA RESPONDER A LA } \\
\text { PREGUNTA }\end{array}$ & $\begin{array}{l}\text { PREGUNTA Y } \\
\text { OPCIONES DE } \\
\text { RESPUESTA } \\
\end{array}$ & $\begin{array}{l}\text { COMENTARIOS SOBRE } \\
\text { EL TIPO DE INFERENCIA }\end{array}$ \\
\hline $\begin{array}{l}\text { Nivel A1 } \\
\text { Tarea } 1 \\
20 \text { de mayo } \\
\text { de } 2011\end{array}$ & $\begin{array}{c}\text { “iHola María! } \\
\text { Te escribo desde Málaga. Mi } \\
\text { empresa necesita un informe } \\
\text { sobre el turismo en el sur de } \\
\text { España y voy a pasar un mes } \\
\text { aquí. Es un trabajo difícil } \\
\text { porque no hablo muy bien } \\
\text { español y utilizo mucho el } \\
\text { diccionario... Volveré a } \\
\text { Francia el día treinta de } \\
\text { este mes... Por las } \\
\text { mañanas, me levanto a las } \\
\text { ocho, desayuno en el hotel } \\
\text { y, después, salgo a trabajar. } \\
\text { Cada día visito una parte } \\
\text { diferente de la costa...” }\end{array}$ & $\begin{array}{l}\text { 1. Pierre escribe un } \\
\text { correo electrónico } \\
\text { sobre... } \\
\text { a) sus vacaciones en } \\
\text { la playa. } \\
\text { b) su trabajo en } \\
\text { Francia. } \\
\text { c) su curso de } \\
\text { español en Málaga. } \\
\text { d) su trabajo en el } \\
\text { sur de España. }\end{array}$ & $\begin{array}{l}\text { 1. Si Pierre está } \\
\text { escribiendo un informe, } \\
\text { está trabajando. } \\
\text { 2. Pierre está escribiendo } \\
\text { un informe. } \\
\text { 3. Por tanto, Pierre está } \\
\text { trabajando. } \\
\text { 4. Si Pierre está en } \\
\text { Málaga, está en el sur de } \\
\text { España. } \\
\text { 5. Pierre está en Málaga. } \\
\text { 6. Pierre está en el sur de } \\
\text { España. } \\
\text { 7. Por tanto, Pierre está } \\
\text { trabajando en el sur de } \\
\text { España. }\end{array}$ \\
\hline $\begin{array}{l}\text { Nivel A2 } \\
\text { Tarea } 2 \\
20 \text { de } \\
\text { noviembre } \\
\text { de } 2010\end{array}$ & $\begin{array}{l}\text { "Hace ya días que quiero } \\
\text { hacer una cena para } \\
\text { enseñaros mi casa a todos } \\
\text { los del grupo. Este fin de } \\
\text { semana estoy muy } \\
\text { ocupada, pero he decidido } \\
\text { hacerla el próximo martes a } \\
\text { las } 21: 30 . \\
\text { ¿Puedes venir? } \\
\text { Había pensado hacerla en } \\
\text { el jardín, pero parece que }\end{array}$ & $\begin{array}{l}\text { 11. La cena será... } \\
\text { a) este fin de } \\
\text { semana. } \\
\text { b) la próxima } \\
\text { semana. } \\
\text { c) en el jardín. }\end{array}$ & $\begin{array}{c}\text { Pregunta } 11 . \\
\text { La respuesta correcta es } \\
\text { la opción b), como } \\
\text { muestra la siguiente } \\
\text { deducción: } \\
\text { 1. El "próximo martes" } \\
\text { implica la "próxima } \\
\text { semana”. } \\
\text { 2. La cena será el } \\
\text { "próximo martes". }\end{array}$ \\
\hline
\end{tabular}


va a llover, así que mejor cenamos en el salón.”

"Ahora Ana trabaja menos, por eso puede hacer una de las cosas que más le

\section{Nivel B1 \\ Tarea 2 23 de agosto de 2013}

\section{Nivel B2 \\ Tarea 1 \\ 23 de \\ agosto de 2013}

$$
\text { gustan: ir al gimnasio muy }
$$
temprano; también le interesa ir al cine, le encanta ver exposiciones de pintura y, lo más importante, pasar su tiempo libre con su familia y sus amigos."
"Fue una experiencia divertida en la que se mezclaban varias cosas: el compañerismo, la competitividad y la extraña sensación de poder realizar esta actividad como parte del trabajo", recuerda Eduardo Loyola, directivo de la consultoría Interface. Loyola fue uno de los conejillos de Indias de un programa diseñado por Óscar de las Mozas, coautor del estudio.

"Entrenábamos fuera del horario laboral. Al

$$
\begin{aligned}
& \text { 12. A Ana le } \\
& \text { gusta... }
\end{aligned}
$$

a) hacer deporte por la mañana.

b) pintar en su tiempo libre.

c) ir al cine con su familia.
C. La cena será la "próxima semana". Pregunta 12

La respuesta correcta es la opción a), como muestra la siguiente deducción:

1. Si a Ana le gusta ir al gimnasio muy temprano,

a Ana le gusta hacer deporte por la mañana. 2. A Ana le gusta ir al gimnasio muy temprano. $C$. A Ana le gusta hacer deporte por la mañana. principio, a algunos compañeros les resultó complicado (sentían vergüenza de verse con indumentaria deportiva), pero pronto nos encontramos trotando por el monte o por pistas de atletismo sin ningún problema. Creo que tener una hora de entrenamiento con los compañeros favorece el trabajo en equipo", dice Loyola.

\section{“Teleplán.}

... En cumplimiento de la normativa vigente nos ponemos en contacto con usted para reiterarle los derechos que le asisten como usuario de nuestros servicios. Tales derechos, detallados a continuación, le serán comunicados
3. En el texto

Eduardo Loyola dice que...

a) se sentía raro

haciendo deporte.

b) el programa

incluía deporte al aire libre.

c) gracias al entrenamiento rendía más.
Pregunta 3

La respuesta correcta es la opción b), como muestra la siguiente deducción:

1. Si Eduardo Loyola trota por el monte o por pistas de atletismo, el programa incluye deporte al aire libre. 2. Eduardo Loyola trota por el monte o por pistas de atletismo.

C. El programa incluye deporte al aire libre.
Tarea 1

19 de noviembre de 2011

\section{Según este documento, el cliente de Teleplán...}

a) puede descargar su contrato desde la página web de la compañía.

b) recibirá comunicación
La respuesta correcta es la opción c), como muestra la siguiente deducción:

\section{Si el cliente de}

Teleplán puede darse de alta telefónicamente, puede contratar los servicios sin firmar ningún contrato. 


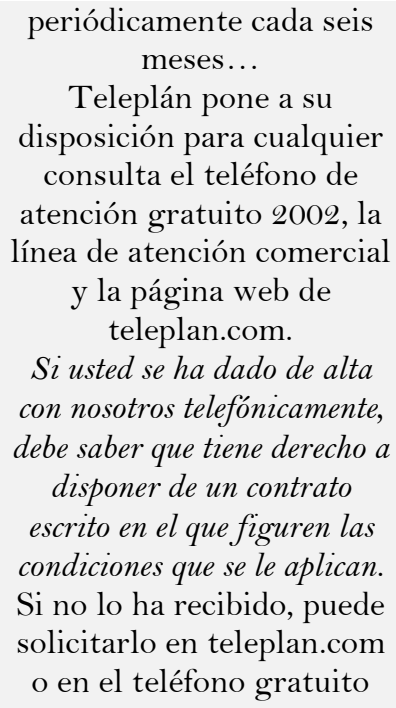

2. El cliente de Teleplán puede darse de alta telefónicamente.

C. El cliente de Teleplán puede contratar sus servicios sin firmar ningún contrato.

Nota: La respuesta correcta la conocemos porque se ofrece en las "Claves" del examen disponibles en el sitio web, pero el comentario sobre el tipo de inferencia solicitada es nuestro. Omitimos un ejemplo del nivel $\mathrm{C}_{2}$ porque no encontramos una tarea de opción múltiple en la prueba administrada el 19 de noviembre de 2011. Asimismo, el énfasis en los textos de la segunda columna es nuestro y lo usamos para realzar en qué fragmento se rastrea la deducción.

Fuente: Instituto Cervantes (2017).

A partir del análisis anterior podemos notar que los reactivos parecen solicitar un tipo de inferencia deductiva. Ejercicios como "Pierre escribe un correo electrónico sobre..." o "La cena será...”, en conjunto con la información que aparece en la columna dos muestran que estamos frente a deducciones, porque la información está completa y la conclusión, que en este caso es la respuesta correcta, no agrega nada nuevo a lo que ya está expresado en las premisas, es decir, la información de la columna dos. Y aunque no estamos afirmando que todas las pruebas de comprensión lectora requieren inferencias de corte deductivo, es interesante apreciar que este tipo de ejercicios son ejemplares, lo cual nos abre el camino para avanzar una propuesta que presentamos a continuación.

\section{Inferencia extendida y racionalidad acotada}

Es comprensible que en las pruebas de comprensión lectora se soliciten únicamente inferencias deductivas porque la deducción es una inferencia clásica que además es viable en términos de diseño. Sin embargo, esta no es una suposición inocente puesto que, como hemos visto en la sección anterior, la deducción no es la única inferencia que tenemos disponible y el enfoque clásico implica ciertos compromisos sobre nuestros criterios de racionalidad.

En efecto, las inferencias deductivas suponen exigencias muy altas. Por ejemplo, como argumenta Morado (2004), las inferencias deductivas parecen implicar que para que una persona sea racional, ésta debería tener tres características: 1) inferir fuera de contexto, como si las inferencias ocurrieran fuera del tiempo y el espacio; 2) tener recursos ilimitados y ser lógicamente omnisciente, como si la persona racional nunca admitiera contradicciones con las consecuencias lógicas de sus creencias; y 3) ser infalible y 
consistente, como si nunca hubiera una necesidad de revisar las creencias pasadas o como si tuviéramos la garantía de que no habrá contradicciones si partimos de premisas consistentes. No obstante, como argumenta Morado, este ideal no corresponde con la realidad, lo cual sugiere que deberíamos adoptar una visión más generosa de inferencia: "Necesitamos un nuevo enfoque que considere de manera rigurosa, y si es posible formal, la estructura de la racionalidad en situaciones concretas, limitadas y falibles" (Morado, 2004, p. 318).

Todd y Gigerenzer (2000), por ejemplo, asumen una perspectiva descriptiva del comportamiento humano y argumentan que para entender la toma de decisiones y el razonamiento en el mundo real (es decir, el mundo de las personas que no tienen poderes sobrenaturales para razonar, ni conocimientos ilimitados, ni tiempo interminable (cf. Fonseca Patrón, 2016) es necesario comprender la noción de racionalidad acotada (bounded rationality), como fue entendida por Herbert Simon, y que puede ser caracterizada por las limitaciones de la mente humana y la estructura de los entornos en los que ésta opera. Dada esta noción de racionalidad acotada, Todd y Gigerenzer (2000) emplean el término de "racionalidad ecológica" para hablar de heurísticas ecológicas: una estrategia de resolución de problemas es ecológicamente racional en la medida en que se adapta a la estructura de un entorno (Todd y Gigerenzer, 2000).

Pero si las normas de racionalidad acotada y la necesidad de una inferencia extendida parecen razonables y, como hemos visto en el apartado anterior, parece que es normal que las pruebas de comprensión lectora utilicen inferencias de tipo deductivo, no resulta incómodo sugerir que el desempeño de los estudiantes en las pruebas de comprensión lectora inferencial podría verse afectado, porque los estudiantes son agentes con racionalidad acotada que llevan a cabo inferencias contextuales. Volveremos a esta propuesta después de mostrar los resultados experimentales.

\section{Metodología}

\subsection{Muestra}

Los participantes fueron $n=187$ universitarios (102 mujeres y 85 hombres), pertenecientes a tres universidades distintas. La edad promedio fue de 19.5 años $(\mathrm{SD} \pm 1,5)$. Al momento de la aplicación del instrumento, todos los participantes eran estudiantes de licenciaturas y semestres diversos, inscritos en cuatro áreas de formación académica, a saber: de Ciencias Económico-Administrativas: 54; de Ingeniería y Ciencias Exactas: 40; de Ciencias Sociales y Humanidades: 39; y de Ciencias Naturales y de la Salud: 54.

\subsection{Instrumento}

Nuestro instrumento es una prueba de comprensión lectora de opción múltiple compuesta por 12 reactivos correspondientes al producto de tres tipos de inferencia (deducción, inducción, abducción), dos tipos de longitud (corto, largo) y dos tipos de complejidad (simple, complejo). Cada reactivo tenía una pregunta asociada a cuatro opciones de respuesta (una sola correcta). A continuación se presentan tres reactivos de la prueba a manera de ejemplo (cuadro 2). 
Cuadro 2. Ejemplos de reactivos del instrumento

\begin{tabular}{|c|c|c|c|}
\hline $\begin{array}{l}\text { NOTAS SOBRE EL } \\
\text { REACTIVO }\end{array}$ & CUERPO DEL TEXTO & $\begin{array}{c}\text { PREGUNTA Y } \\
\text { OPCIONES DE } \\
\text { RESPUESTA }\end{array}$ & $\begin{array}{l}\text { JUSTIFICACIÓN DE LA } \\
\text { RESPUESTA CORRECTA }\end{array}$ \\
\hline $\begin{array}{c}\text { Reactivo 1 } \\
\text { Referencia del } \\
\text { texto: } \\
\text { Martínez Rica, J. } \\
\text { P. (1979). } \\
\text { Enciclopedia de la } \\
\text { vida animal. } \\
\text { México: Bruguera } \\
\text { Mexicana de } \\
\text { ediciones, S.A. } \\
\text { Tomo 13, p. 1907. } \\
\text { Texto largo y } \\
\text { simple. }\end{array}$ & $\begin{array}{l}\text { El pájaro bobo real (o } \\
\text { pingüino) "no } \\
\text { construye un nido, sino } \\
\text { que guarda su único } \\
\text { huevo sobre sus pies, } \\
\text { protegiéndolo con un } \\
\text { repliegue de la piel } \\
\text { abdominal. Sin } \\
\text { embargo, permanece } \\
\text { en un pequeño } \\
\text { territorio en vez de ir y } \\
\text { venir con el huevo. La } \\
\text { puesta se inicia en } \\
\text { noviembre y dura } \\
\text { hasta abril. Después de } \\
\text { confiar el huevo al } \\
\text { macho, la hembra se } \\
\text { ausenta por dos } \\
\text { semanas en busca de } \\
\text { comida, y de este modo } \\
\text { rehace las reservas } \\
\text { alimenticias perdidas al } \\
\text { formar el huevo. En } \\
\text { adelante, ambos } \\
\text { progenitores se } \\
\text { turnarán en el cuidado } \\
\text { del huevo o polluelo.” }\end{array}$ & $\begin{array}{l}\text { De acuerdo al texto } \\
\text { anterior, qué se } \\
\text { concluye } \\
\text { necesariamente: } \\
\text { A) que el pájaro bobo } \\
\text { vive y muere en un } \\
\text { pequeño territorio. } \\
\text { B) que la incubación } \\
\text { del pájaro bobo real } \\
\text { dura seis meses. } \\
\text { C) que el macho del } \\
\text { pájaro bobo real no } \\
\text { come hasta abril. } \\
\text { D) que algún pájaro } \\
\text { bobo real es buen } \\
\text { constructor de nidos. }\end{array}$ & 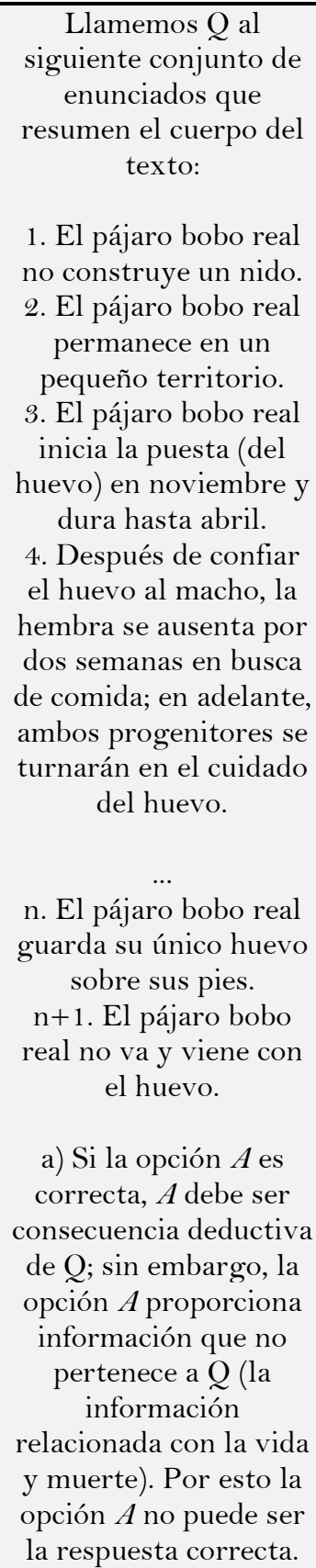 \\
\hline & & & $\begin{array}{l}\text { b) Si la opción } B \text { es } \\
\text { incorrecta, } B \text { no debe } \\
\text { ser consecuencia } \\
\text { deductiva de } Q ; \text { sin } \\
\text { embargo, de } 3 \text { se sigue } \\
\text { que, si el pájaro bobo } \\
\text { real inicia la puesta } \\
\text { (del huevo) en } \\
\text { noviembre y dura } \\
\text { hasta abril, entonces la }\end{array}$ \\
\hline
\end{tabular}




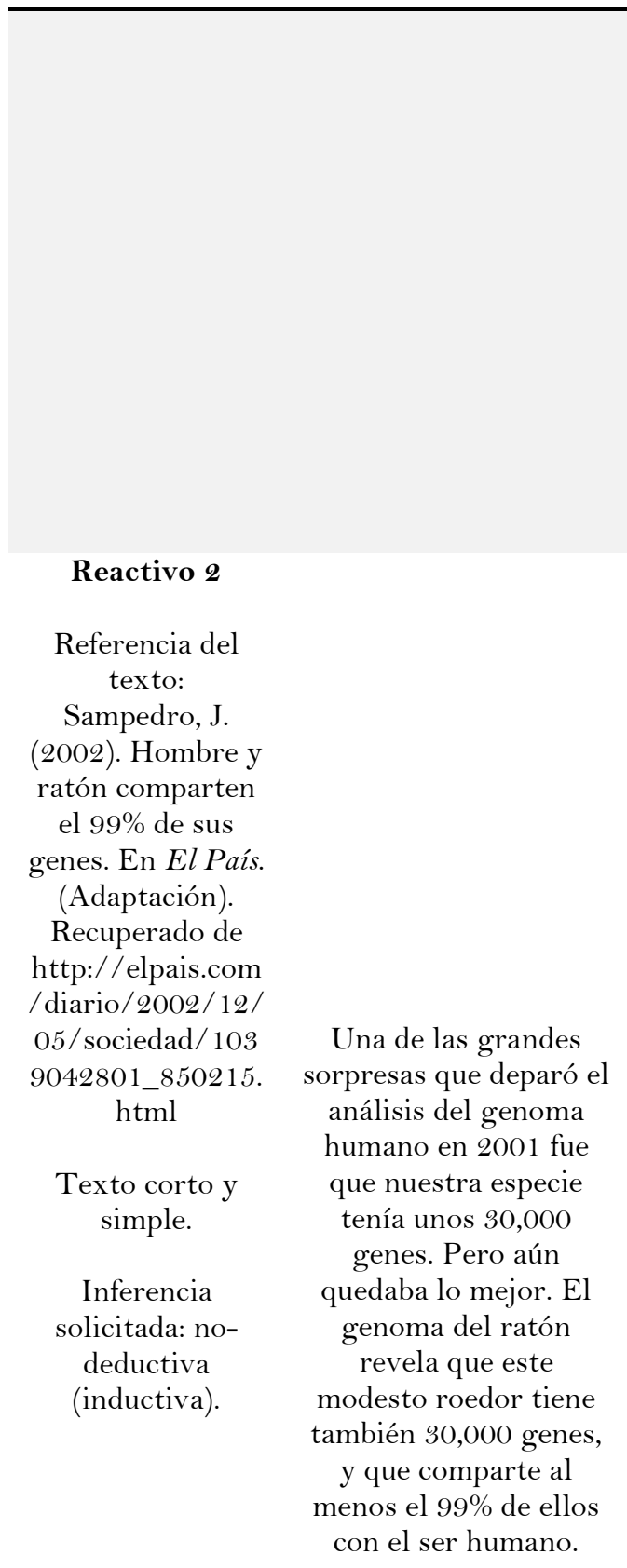

De acuerdo al texto anterior, qué es lo más probable que hagan los científicos en el futuro:

A) estudiarán las diferencias entre los seres humanos y los ratones.

B) invertirán en la crianza de ratones de laboratorio de forma masiva.

C) podrán estudiar casi todas las enfermedades humanas en el ratón. D) se especializarán en las características fisiológicas de los ratones. incubación del pájaro bobo real dura seis meses, que es justamente una paráfrasis de $B$. Por esto la opción $B$ es la respuesta correcta.

c) La opción $C$ no es la respuesta correcta, como en el inciso a).

d) La opción $D$ no es la respuesta correcta, como en el inciso a). Para justificar la corrección de este reactivo, les solicitamos a cinco lectores expertos que lo resolvieran, ellos por probabilidad subjetiva eligieron como respuesta más frecuente la opción C. Esta elección significaría que la conclusión más probable a partir del cuerpo del texto (en el que se enuncia la gran similitud genética entre los seres

humanos y los ratones) es la opción C. De hecho, al conocerse tal similitud, parece

altamente probable que se realicen distintos experimentos en el ratón que para el caso de los seres humanos no sería éticamente permitido; de esta

forma, lo más probable que harían los

científicos en el futuro sería que podrían estudiar casi todas las enfermedades humanas en el ratón.

Si bien las opciones A, B y D son probables, no representan la opción más probable en términos de lo que se sabe en la actualidad 
sobre este tipo de

experimentos.

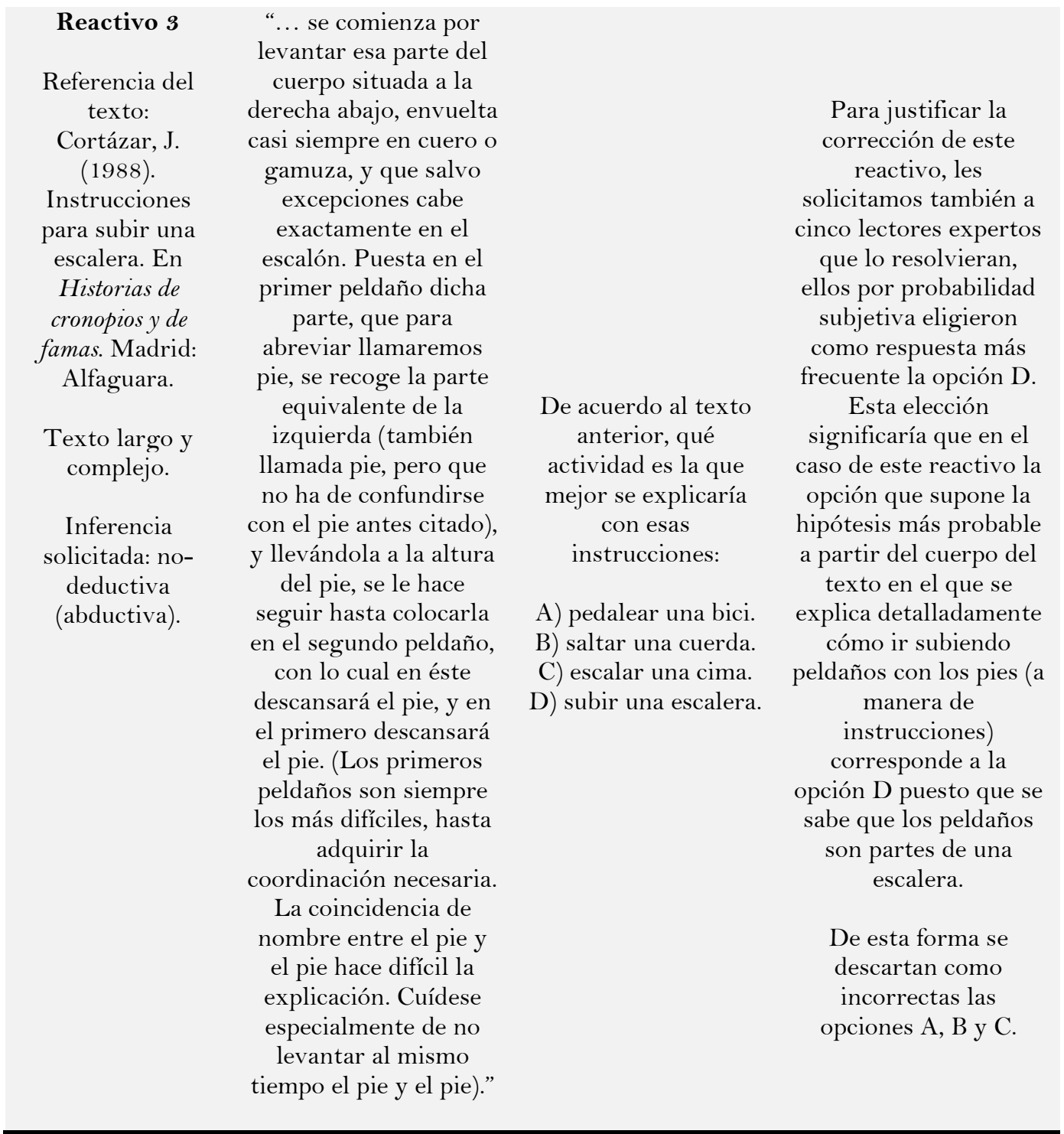

Fuente: Elaboración propia.

\subsection{Procedimiento}

Se les solicitó a los estudiantes que firmaran una carta de consentimiento informado donde se les explicaba la confidencialidad con la que se conservarían sus datos personales. Posteriormente, se les leyeron las instrucciones de la prueba de forma grupal. Durante la prueba se les midió el tiempo, de tal manera que los estudiantes no excedieran 40 minutos para responderla. Las condiciones para resolver la prueba fueron favorables en relación al horario matutino y al lugar (salones de clase).

\subsection{Análisis estadístico}

El análisis se realizó con el paquete estadístico R versión 2.8.1 (R Development Core Team, 2008). Para establecer si la probabilidad de contestar correctamente a un reactivo estuvo afectada por la longitud, la complejidad y el tipo de inferencia utilizamos un modelo 
lineal generalizado mixto (GLMM) con una distribución binomial y una función de enlace logit; en él incluimos, como variable dependiente, la probabilidad de responder correctamente; y como variables independientes: la longitud (factor con dos niveles: corto y largo), la complejidad (factor con dos niveles: simple y complejo) y el tipo de inferencia (factor con tres niveles: deductivo, inductivo y abductivo). Además, el modelo incluyó todas las interacciones entre estas tres variables. Consideramos, también, la identidad del participante como variable aleatoria debido a que los datos no son independientes entre sí y realizamos selección de modelos mediante remoción secuencial de términos que no tuvieran una significancia estadística.

\section{Resultados}

Encontramos un efecto de la longitud, la complejidad y el tipo de inferencia sobre la probabilidad de tener la respuesta correcta. En el cuadro 3 presentamos los estadísticos que permiten apreciar con claridad los valores de significancia entre las variables de interés.

Cuadro 3. Efecto de la longitud, la complejidad y el tipo de inferencia sobre la probabilidad de contestar correctamente

\begin{tabular}{lcccc}
\hline \multicolumn{1}{c}{ TÉRMINOS } & COEFICIENTE & $\begin{array}{c} \pm \text { ERROR } \\
\text { ESTÁNDAR }\end{array}$ & $\boldsymbol{\chi}^{2}$ & P \\
\hline Inferencia*longitud**omplejidad & $-1,935$ & 0,226 & 79,004 & $<0,001$ \\
Inferencia*longitud & $-0,329$ & 0,105 & 9,944 & 0,002 \\
Inferencia*complejidad & $-0,534$ & 0,104 & 26,842 & $<0,001$ \\
Longitud & 0,817 & 0,053 & 71,844 & $<0,001$ \\
Complejidad & 0,252 & 0,097 & 6,790 & 0,009 \\
Inferencia & 0,941 & 0,051 & 403,360 & $<0,001$ \\
\hline
\end{tabular}

Nota: Los asteriscos indican la interacción de los términos (variables) considerados en cada análisis. Las filas muestran los términos que no fueron removidos (remoción secuencial) por su significancia estadística. La única interacción rechazada fue: Longitud*complejidad.

Fuente: Elaboración propia.

Tomando la complejidad del texto como la perspectiva desde la cual describiremos los resultados, observamos, por un lado, que en las preguntas que solicitaban una inferencia deductiva, cuando el texto era complejo y corto, 63 estudiantes contestaron correctamente, lo que equivale a una probabilidad de 0,34; en contraste, con un texto complejo y largo, únicamente 40 estudiantes contestaron correctamente, lo que equivale a una probabilidad de 0,21 . Ahora bien, en las preguntas que requerían una inferencia abductiva, cuando el texto era complejo y largo, 124 estudiantes contestaron correctamente, lo que equivale a una probabilidad de 0,66 ; en contraste, con un texto complejo y corto, únicamente 38 estudiantes contestaron correctamente, es decir, hay una probabilidad de 0,2. Por último, en las preguntas que promovían una inferencia inductiva, cuando el texto era complejo y largo, 176 estudiantes contestaron correctamente, lo que equivale a una probabilidad de 0,94 ; en contraste, con un texto complejo y corto, 157 estudiantes contestaron correctamente, lo que equivale a una probabilidad de 0,84. En suma, con textos complejos y largos, los estudiantes tienen más respuestas correctas si el texto solicita una inferencia no-deductiva. 
Observamos, por otro lado, que en las preguntas que demandaban una inferencia deductiva, cuando el texto era simple y largo, 135 estudiantes contestaron correctamente, lo que significa una probabilidad de 0,72 ; en contraste, con un texto simple y corto, únicamente 29 estudiantes contestaron correctamente, lo que equivale a una probabilidad de 0,16 . Ahora bien, en las preguntas que solicitaban una inferencia abductiva, cuando el texto era simple y corto, 96 estudiantes contestaron de manera correcta, con una probabilidad de 0,51; en contraste, con un texto simple y largo, 45 estudiantes contestaron correctamente, lo que equivale a una probabilidad de 0,46. Por último, en las preguntas que pedían una inferencia inductiva, cuando el texto era simple y largo, 154 estudiantes contestaron correctamente, lo cual es una probabilidad de 0,82 que coincide con los resultados de cuando el texto era simple y corto, donde 153 estudiantes contestaron correctamente. En suma, con textos simples y largos, los estudiantes tienen más respuestas correctas si el texto solicita una inferencia inductiva, pero con textos simples y cortos, los estudiantes tienen menos respuestas correctas si el texto solicita una inferencia deductiva. En la figura 1 presentamos visualmente los resultados previamente descritos.

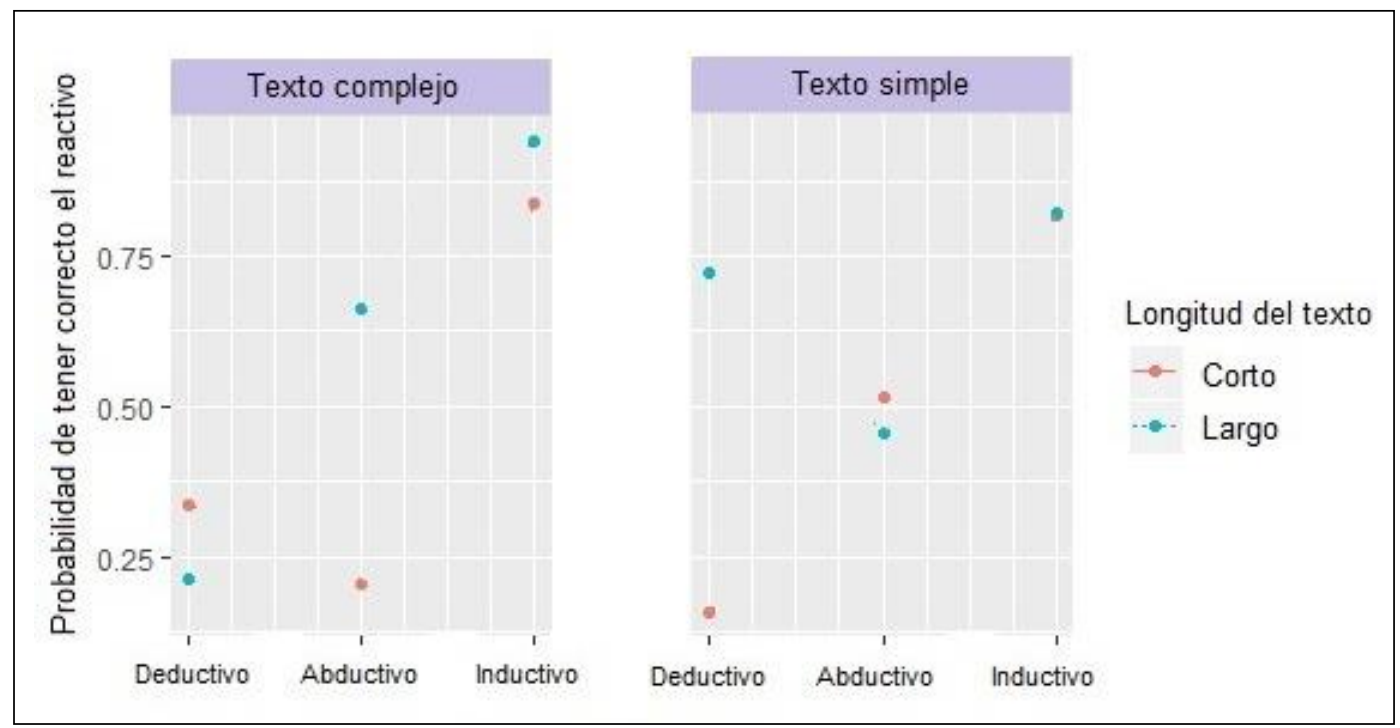

Figura 1. Efecto de la longitud, complejidad y tipo de inferencia sobre la probabilidad de responder correctamente.

Fuente: Elaboración propia.

\section{Discusión y conclusiones}

\subsection{Discusión}

Como se ha referido anteriormente, la comprensión lectora es un proceso que no se puede observar directamente (cf. Pérez Zorrilla, 2005; Fletcher, 2006) y por esto, para evaluarla, se suelen realizar pruebas para medir el desempeño de los estudiantes. En este trabajo evaluamos la comprensión lectora inferencial considerando los atributos estructurales de los textos y las propiedades estructurales de las pruebas. De esta forma, asociamos la longitud con la microestructura; la complejidad con la macroestructura; y los tipos de inferencia con la superestructura. 
Con respecto a la longitud (microestructura), notamos que cuando un texto es corto (además de simple y deductivo) sólo el 16\% de los estudiantes responde correctamente, lo cual podría explicarse por la alta densidad de información que posee un texto corto, lo que hace que el texto sea difícil de comprender; al respecto, Pallotti (2013) afirma que una idea errónea que se tiene comúnmente es la de pensar que para que un texto sea más simple baste con acortarlo, cuando en realidad, si se mantiene constante la cantidad de información, un texto más corto será más denso en términos de la información que presenta (en comparación con un texto largo) y la densidad conceptual es, por ello, una de las principales causas de dificultad.

Con respecto a la complejidad (macroestructura) observamos que cuando un texto es complejo (además de largo e inductivo) el 94\% de los estudiantes lo responde correctamente, lo cual podría explicarse de la siguiente manera: cuando un estudiante se enfrenta a un texto complejo éste debe comprenderlo mediante su cohesión y coherencia (Ferrari, 2003), lo cual podría llevarlo a realizar relecturas más atentas en contraste con textos simples que lo llevarían a realizar lecturas menos atentas.

Con respecto a la inferencia (superestructura) notamos dos datos particularmente interesantes: 1) cuando un texto promueve una inferencia deductiva (además de simple y largo), el $72 \%$ de los estudiantes lo responde correctamente, lo que permite argumentar a favor de la presencia continua de textos deductivos en pruebas de comprensión lectora, lo cual es consistente con los diseños usuales como hemos visto, por ejemplo, a partir del análisis de la Tabla 1; pero también, 2) cuando un texto promueve una inferencia nodeductiva, específicamente inductiva, (además de ser complejo y corto), el $84 \%$ de los estudiantes lo responde correctamente, lo que nos permite argumentar a favor de la inclusión de tex tos no-deductivos (inductivos) en pruebas de comprensión lectora. Esto es consistente con los conceptos de racionalidad acotada e inferencia extendida: en efecto, el desempeño de los estudiantes se ve favorecido cuando incluimos un criterio más generoso de inferencia, lo cual está en sintonía con un concepto de racionalidad acotada.

\subsection{Conclusiones}

Es razonable concluir que las características de microestructura (longitud), macroestructura (complejidad) y superestructura (inferencia) de una prueba de comprensión lectora inferencial influyen en el desempeño de los estudiantes, por lo cual resulta plausible reconsiderar el uso de inferencias no-deductivas en el diseño de pruebas de comprensión lectora, pues esto podría fortalecer los instrumentos de evaluación que utilizamos para medir el nivel de comprensión lectora de nuestros estudiantes.

Además, sabiendo que estas pruebas pretenden medir el grado de interacción entre el lector y el texto, podemos mencionar que las características del texto que hemos estudiado (longitud, complejidad e inferencia) están relacionadas con características del lector que hemos dado por supuestas. Por ejemplo, cuando un estudiante se encuentra inscrito en un programa de licenciatura en una universidad, posee un conjunto de conocimientos aprendidos durante los cursos en los niveles escolares inferiores. Estos conocimientos previos al momento de resolver una prueba de comprensión lectora inferencial ofrecen elementos a los estudiantes para obtener conclusiones a partir de enunciados en los cuales estas conclusiones no se encuentran referidas explícitamente. Incluso, un estudio futuro podría comparar el desempeño de los estudiantes en relación a su área de estudio de procedencia. 
Por último, es necesario comentar que, debido a restricciones temporales, una limitación de este estudio fue el uso de 12 reactivos únicos para agotar todas las combinaciones posibles de las variables de interés sin repetición, por lo que, en trabajos futuros, podríamos estudiar el desempeño de los estudiantes con más reactivos para garantizar no sólo todas las combinaciones sino también repetición. Además, valdría la pena llevar a cabo una investigación similar a la que hemos presentado aquí, pero considerando, además de las propiedades estructurales de la prueba, las características de los estudiantes en términos de sus estrategias de lectura o de resolución de problemas (cf. Platas-García, Castro-Manzano y Reyes-Meza, 2016). Asimismo, parece muy conveniente que en estudios futuros se comparara el desempeño de los estudiantes en dos tipos de pruebas, a saber: una con preguntas únicamente deductivas y otra con preguntas deductivas y nodeductivas.

Agradecimientos. Nos gustaría agradecer a los evaluadores por sus precisas correcciones y útiles comentarios. Este trabajo fue parcialmente financiado por el proyecto UPAEP 30108-1008.

\section{Referencias}

Acosta, I. (2009). La comprensión lectora, enfoques y estrategias utilizadas durante el proceso de aprendizaje del idioma español como segunda lengua. Tesis doctoral. Universidad de Granada, España.

Aliseda, A. (1997). Seeking Explanations: Abduction in Logic, Philosophy of Science and Artificial Intelligence. Amsterdam: Institute for Logic, Language and Computation, Universidad de Amsterdam.

Aliseda, A. (1998). La abducción como cambio epistémico: C. S. Peirce y las teorías epistémicas en inteligencia artificial. Analogía, 12, 125-144.

Anderson, R. C. y Pearson, P. D. (1988). A schema-theoretic view of basic processes in reading comprehension. En P. Carrell, J. Devine, D. Eskey. (Eds.), Interactive approaches to second language reading (pp. 37-55). Cambridge: Cambridge University Press.

Bell, T. (2001). Extensive reading: speed and comprehension. The Reading Matrix, 1(1). Recuperado de http://www.readingmatrix.com/articles/bell/

Consejo de Europa. (2002). Marco común europeo de referencia para las lenguas: aprendizaje, enseñanza, evaluación. Estrasburgo: Consejo de Europa, Ministerio de Educación y Grupo Anaya.

Cook, R. T. (2009). A Dictionary of Philosophical Logic. Edimburgo: Edinburgh University Press.

Copi, I. y Cohen, C. (2007). Introducción a la Lógica. Ciudad de México: Limusa.

Ferrari, S. (2003). Comprensione e lettura in L2: uno studio sperimentale sugli effetti della modificazione testuale. En R. Grassi, A. Valentini y R. Bozzone Costa. (Eds.), L'italiano per lo studio nella scuola plurilingue: tra semplificazione e facilitazione (pp. 85-110). Perugia: Guerra.

Fletcher, J. M. (2006). Measuring reading comprehension. Scientific Studies of Reading, 10, 323-330.

Fonseca Patrón, A. L. (2016). El debate sobre las heurísticas. Una disputa sobre los criterios de buen razonamiento entre la Tradición de Heurística y Sesgo y la Racionalidad Ecológica. Valenciana, 17, 87-115. 
García, E. (1993). La comprensión de textos. Modelo de procesamiento y estrategias de mejora. Didáctica (Lengua y literatura), 5, 87-114.

Giovannini, M. L. y Rosa, A. (2015). Prove standardizzate di comprensione dei testi per la scuola secondaria di primo grado II. In uscita dalla classe seconda e in entrata nella classe terza. Milano: LED.

Guerra, J. y Guevara, Y. (2013). Validación de un instrumento para medir comprensión lectora en alumnos universitarios mexicanos. Enseñanza e Investigación en Psicología, 18(2), 277-291.

Guerra, J. y Guevara, C. Y. (2017). Variables académicas, comprensión lectora, estrategias y motivación en estudiantes universitarios. Revista Electrónica de Investigación Educativa, 19(2), 78-90.

Hawthorne, J. (2014). Inductive Logic. Recuperado de http://plato.stanford.edu/archives/win2014/entries/logic-inductive

Instituto Cervantes. (2017). Exámenes DELE. Preparar la prueba DELE. Recuperado de https://examenes.cervantes.es/es/dele/preparar-prueba

Intraversato, A. (2013). La comprensione della lettura fra abilità e conoscenze enciclopediche. Indagine sui Licei. Roma: Ediciones Nuova Cultura.

Mangen, A., Walgermo, B. R. y Brønnick, K. (2013). Reading linear texts on paper versus computer screen: Effects on reading comprehension. International Journal of Educational Research, 58, 61-68. https://doi.org/10.1016/j.ijer.2012.12.002

Morado, R. (2004). Problemas filosóficos de la lógica no monotónica. En R. Orayen y A. Moretti (Eds.), Filosofía de la lógica, Enciclopedia Iberoamericana de Filosofía (pp. 313-344). Madrid: Trotta y Consejo Superior de Investigaciones Científicas.

Okasha, S. (2002). Philosophy of Science: A very Short Introduction. Oxford: Oxford University Press.

Pallotti, G. (2013). Aiutare a capire e ad apprendere. En F. Pagnozzi y P. Weber (Eds.), Testi narrativi per le classi con competenze linguistiche diversificate. Italiano come lingua seconda (pp. 355). Trento: IPRASE.

Pérez Zorrilla, M. J. (2005). Evaluación de la comprensión lectora: dificultades y limitaciones. Revista de Educación, Ext., 121-138.

Platas-García, A., Castro-Manzano, J. M. y Reyes-Meza, V. (2016). Razonamiento y heurísticas en pruebas de comprensión lectora. Revista de Educación, 371(1), 157-187. https://doi.org/10.4438/1988-592X-RE-2015-371-312

R Development Core Team. (2008). A Language and Environment for Statistical Computing. Viena: R Foundation for Statistical Computing.

Rice, G. E. (1994). Examining constructs in reading comprehension using two presentation modes: Paper vs. computer. Journal of Educational Computing Research, 11(2), 153-178. https://doi.org/ 10.2 190/MV46-VW49-4G5W-FM92

Rodríguez Rodríguez, R. (2005). Abducción en el contexto del descubrimiento científico. Revista de Filosofía de la Universidad de Costa Rica, 43(109/110), 87-97.

Salazar, L. (2006). Interdependencia lingüística, transferencia y enseñanza-aprendizaje de lenguas extranjeras. Laurus, 12, 45-72.

Sánchez Lobato. J. (Coord.). (2007). Saber escribir. Madrid: Aguilar/Instituto Cervantes. 
Serrano, M. A., Vidal-Abarca, E. y Ferrer, A. (2017). Decisiones estratégicas de lectura y rendimiento en tareas de competencia lectora similares a PISA. Educación XX1, 20(2), 279297. https://doi.org/10.5944/educXX1.12076

Solé, I. (1987). Las posibilidades de un modelo teórico para la enseñanza de la comprensión lectora. Infancia y Aprendizaje, 39-40, 1-13.

Todd, P. M. y Gigerenzer, G. (2000). Précis of simple heuristics that make us smart. Behavioral y Brain Sciences, 23, 727-780.

Van Dijk, T. A. (1992). La ciencia del texto. Un enfoque interdisciplinario. Barcelona: Paidós Comunicación.

Zambelli, L. (2014). Semplificare i testi di studio: quando, come. Italiano LinguaDue, 1, 327-341.

\section{Breve CV de los autores}

\section{Alejandra Platas-García}

Licenciada en Filosofía y Maestra en Pedagogía. Actualmente estudia el Doctorado en Educación en la Universidad Popular Autónoma del Estado de Puebla (UPAEP). Es docente de italiano como lengua extranjera en el Centro de Lenguas Extranjeras de la Facultad de Lenguas de la Benemérita Universidad Autónoma de Puebla (BUAP). Realiza su investigación sobre la evaluación de la comprensión lectora inferencial a través de pruebas de opción múltiple. Asimismo, se interesa por las estrategias para la resolución de pruebas de comprensión lectora inferencial que emplean los estudiantes universitarios. ORCID ID: 0000-0002-5150-0668. Email: aplatasg@gmail.com

\section{J. Martín Castro-Manzano}

Licenciado en Filosofía y Maestro en Inteligencia Artificial por la Universidad Veracruzana (UV), Doctor en Filosofía de la Ciencia por la Universidad Nacional Autónoma de México (UNAM); tiene estudios en Ciencias Computacionales por la BUAP. Actualmente es docente investigador de la Facultad de Filosofía y Humanidades en la UPAEP. Es miembro del Sistema Nacional de Investigadores (SNI, nivel 1), de la Academia Mexicana de Lógica y de la Association for Symbolic Logic. Realiza su investigación sobre Sistemas formales y Didáctica de la Lógica. ORCID ID: 0000-00032227-921X. Email: josemartin.castro@upaep.mx

\section{Verónica Reyes-Meza}

Licenciada en Psicología y Maestra en Diagnóstico y Rehabilitación Neuropsicológica. Doctora en Neuroetología, realizó un Posdoctorado en el posgrado en Ciencias Biológicas de la Universidad Autónoma de Tlaxcala (UATx). Actualmente es docente investigadora del Centro Tlaxcala de Biología de la Conducta en la UATx. Es miembro del Sistema Nacional de Investigadores (SNI, nivel 1) y de la Society for Neurosciences. Su principal línea de investigación es el análisis experimental de la conducta. ORCID ID: 0000-00022745-4032. Email: veronica.reyesm@uatx.mx 


\section{Irene Gaona-Gordillo}

Licenciada en Biología por la Universidad Nacional Autónoma de México (UNAM) y Maestra en Ciencias Biológicas por la Universidad Autónoma de Tlaxcala (UATx). Es experta en análisis estadístico y programadora en paquetería $R$, específicamente en modelos generalizados con efectos mixtos. Realiza su investigación dentro de las áreas de Biología del Comportamiento y Ecofisiología. Email:r.gago.r@gmail.com 